\title{
Wet Synthesis of Monodisperse Cobalt Oxide Nanoparticles
}

\author{
Taimur Athar, Abdul Hakeem, Neha Topnani, and Ameed Hashmi
}

Organic III, Indian Institute of Chemical Technology, Tarnaka, Hyderabad 500-007, India

Correspondence should be addressed to Taimur Athar, taimurathar2001@gmail.com

Received 31 December 2011; Accepted 19 February 2012

Academic Editors: F. M. Labajos, B. Luan, and M. Padmanaban

Copyright () 2012 Taimur Athar et al. This is an open access article distributed under the Creative Commons Attribution License, which permits unrestricted use, distribution, and reproduction in any medium, provided the original work is properly cited.

\begin{abstract}
Ultrafine and monodispersable colloidal cobalt oxide nanoparticles were successfully synthesized quantitatively via soft chemical approach with controlled particle size and microstructural properties for their use in technological applications. The particle size, shape, and other microstructural properties are directly influenced by their reaction conditions. The FT-IR studies give information for phase purity, and ultraviolet absorption spectroscopy helps to study the optical properties. Thermal analysis gives the information about thermal stability. With the help of X-ray diffraction pattern, the size of the particle was calculated. An electron microscope studies help in morphological characterization, and Brunauer-Emmett-Teller method gives information about surface area. Cobalt oxide nanoparticle tends to orient itself with its narrow size distribution having a crystal size around $50 \mathrm{~nm}$.
\end{abstract}

\section{Introduction}

In recent years, synthesis of transition metal oxide nanoparticles has attracted much attention because of their outstanding multifunctional physical-chemical properties for their use in different fields. The actual challenge that depends on how to optimize a cost-effective synthetic methodology via soft chemical approach that gives technological grade nanomaterials with a specific structural-morphological functional properties remains a challenge to synthetic chemists. Cobalt oxide nanopowder is widely used in many fields such as magnetic [1], gas sensor [2], lithium ion batteries [3], catalysis [4], and electrochemical [5] depending on the size, structure, shape, and phase homogeneity and with surface morphologies. Many approaches were made for the successful synthesis of cobalt oxide nanoparticles in past one decade by using different synthetic approaches, such as thermal method [6], precipitation methods [7], pyrolysis process [8], and sonochemical method [9]. However, all these methods have a limited control in particle functional properties with low yield. Therefore, it is necessary to find alternative method for the synthesis of nanopowder that should be cost-effective and environmental friendly. The soft chemical approach is the best synthetic method which helps to synthesize of cobalt oxide nanopowder. Soft chemistry that helps to increase a functional efficiency for its use in technology also helps in better understanding the crystal growth with a required shape, size, and phase purity by controlling surface energies. We have used wet chemical approach to prepare ultrapure monodisperse tetrapod-shaped cobalt oxide nanoparticles with a narrow size distribution and shape with good crystallinity in quantitative yields which can be used for a largescale production as well as in technological applications.

\section{Experimental}

2.1. Materials Used. AR grade reagents were purchased from Aldrich with $99.5 \%$ purity and used as such. During the experimental procedure, the Millipore water was used. Solvents were obtained from commercial source. Purification of solvents was carried out as reported in a procedure [10].

\footnotetext{
2.1.1. Preparation of Cobalt Oxide Nanoparticles via Soft Chemical Approach. The aqueous solution was prepared by mixing the calculated amount of $\mathrm{KOH}(5.61 \mathrm{~g}, 100 \mathrm{mmol})$ and Cobalt acetate $(7.08 \mathrm{~g}, 40 \mathrm{mmol})$ and then stirred for 2 hours followed by refluxing for 4 hours as shown in Scheme 1. After filtration, the residue was washed with distilled water until the eluent shows $\mathrm{pH}$ 7. The residue was calcinated at $450^{\circ} \mathrm{C}$ for 4 hours in dry nitrogen. Black powder was obtained with $85 \%$ yield.
} 


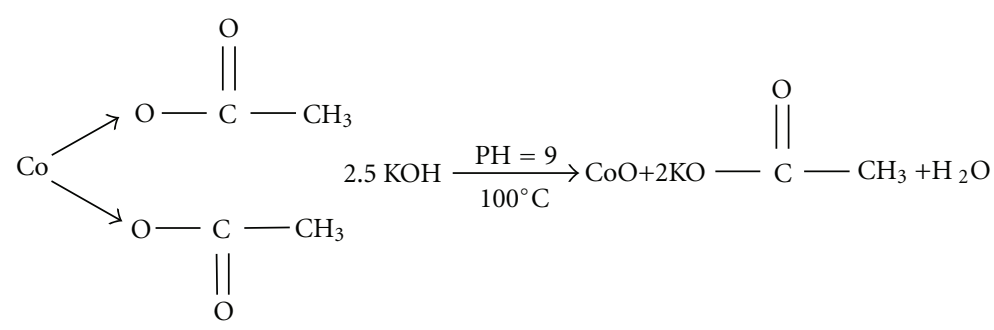

Scheme 1

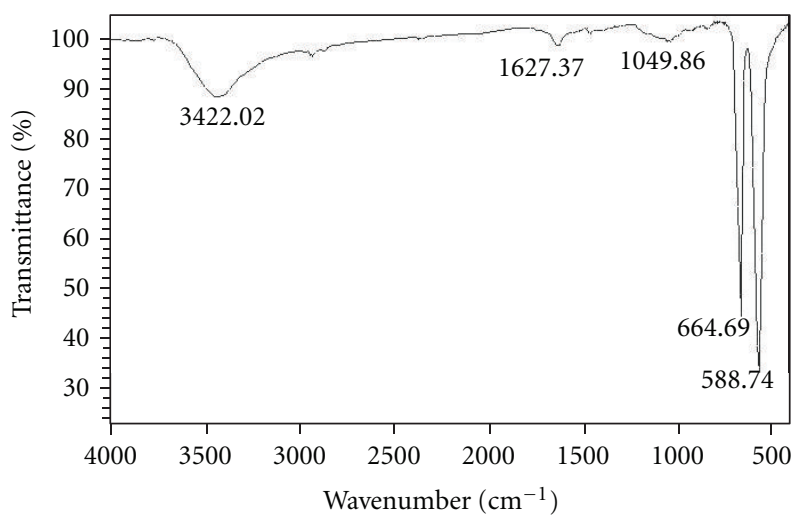

FIGURE 1: FT-IR spectrum was recorded for cobalt oxide nanoparticles after calcination at $450^{\circ} \mathrm{C}$ for 4 hours.

2.1.2. Characterization. Structural and morphological characterization of as-prepared cobalt oxide nanoparticle was carried out with the help of following techniques as discussed herein after. FT-IR studies were carried out at room temperature in the range of 4000 to $400 \mathrm{~cm}^{-1}$ by using $\mathrm{KBr}$ pellets in a Perkin-Elmer Spectrometer GX model. UV-visible diffuse reflectance spectra UV-DRS were recorded on a GBC UV-visible Cintra 10/20/40 spectrometer on dry-pressed disk solid samples using $\mathrm{KBr}$ as dilutor as well as the reference in the range of $200-800 \mathrm{~nm}$ with a scan speed of $20 \mathrm{~nm}$ per minute. The phase purity was determined by thermal transformation pattern as studied by TG-DSC (Mettler Toledo star, Columbus, $\mathrm{OH}$ ) in air, with a heating rate of $10^{\circ} / \mathrm{min}$ from ambient temperature to $1000^{\circ} \mathrm{C}$. X-ray powder diffraction patterns were taken in reflection mode $\mathrm{CuK} \alpha$ $(\lambda=1.5406 \AA)$ radiation in the $2 \theta$ range from $0 \theta$ to $80 \theta$ on a Seimens (Cheshire, UK) D5000X-ray diffractometer by continuous scanning. Scanning electron microscope images help to give surface properties at the room temperature with the help of Hitachi S520 scanning electron microscope. EDX studies help in providing the metal-oxygen ratio with the help of Oxford link ISIS-300 instrument. The morphologies of the powder were further investigated with the help of Philips Tecnai G ${ }^{2}$ FEI F12 Transmission Electron Microscope operated at $80-100 \mathrm{KV}$. The samples for TEM were prepared by loading a sample in hexane-suspension onto a formvarcoated copper grid. The BET surface area analysis was done in AUTOSORB 1t under nitrogen atmosphere after degassing the samples at $200^{\circ} \mathrm{C}$ for $1 \mathrm{~h}$.

\section{Results and Discussion}

The strong corelationship exists between particle size and shape in inorganic materials for their potential application as sensors. Particle properties can be tuned after optimization with the help of reaction time, temperature, $\mathrm{pH}$, type of precursor used, and its concentration.

3.1. FT-IR Studies. Cobalt oxide nanoparticles were synthesized via chemie-douce approach that shows the presence of limited agglomeration-taking place at room temperature due to high surface energies as shown in Figure 1. Annealing at $450^{\circ} \mathrm{C}$ for four hours in the presence of an inert atmosphere leads to the change of amorphous to the crystalline state with narrow particle size distribution with well-defined particle size, shape, and phase purity. A stretching frequency at $3422 \mathrm{~cm}^{-1}$ and a weak asymmetric band at $1627 \mathrm{~cm}^{-1}$ support the presence of $\mathrm{OH}^{-}$group due to the absorption of water by nanoparticle during sample preparation. The presence of two strong $\mathrm{M}-\mathrm{O}$ stretching and bending frequencies at $664.69 \mathrm{~cm}^{-1}$ and $568.74 \mathrm{~cm}^{-1}$, respectively, supports the presence of phase purity with monodispersity in the facecentered cubic structure [6].

3.2. UV-Vis Absorption. An optical property of cobalt oxide nanoparticle was recorded with respect to high purity, and its crystallinity was confirmed with the help of UV-visible absorption spectra by observing an excitonic absorbance 


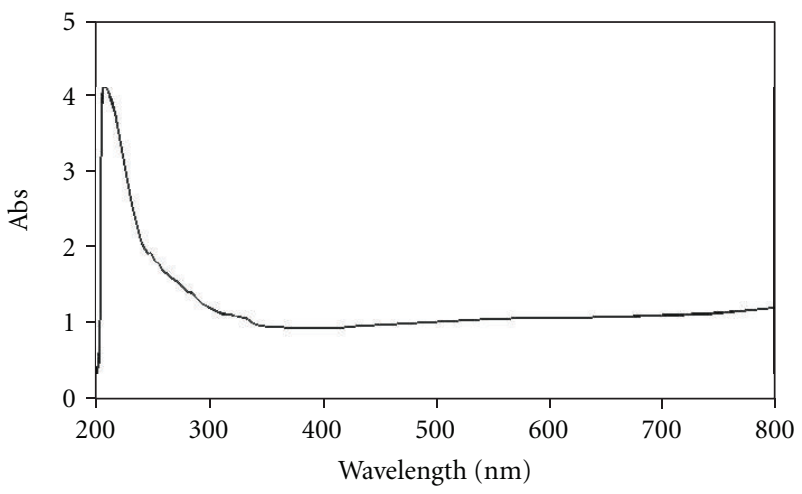

FIGURE 2: UV-Vis absorption spectrum recorded for cobalt oxide nanoparticles after calcination at $450^{\circ} \mathrm{C}$.

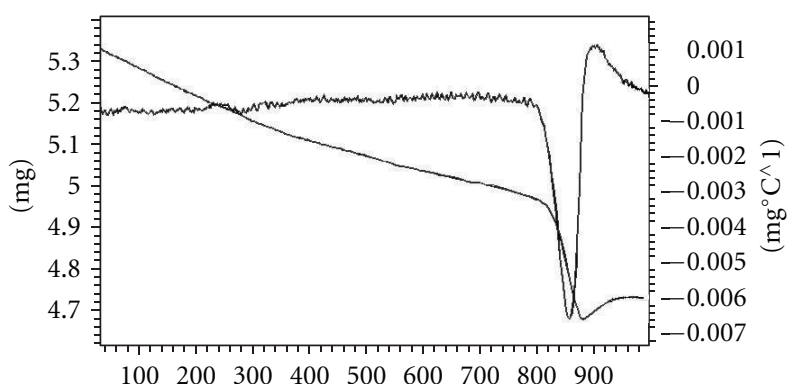

FIGURE 3: TGA and DSC analysis was carried out for cobalt oxide nanoparticles after calcination at $450^{\circ} \mathrm{C}$.

band at $240 \mathrm{~nm}$ with a tail extending towards a longer wavelength due to their quantum size effects as shown in Figure 2 [11]. On ageing no significant absorption peaks were observed probably due to the quantum confinement effects in the energy gap. The absorption peaks exhibit a slightly broad peak due to the particle size. The stability of $\mathrm{CoO}$ nanoparticle can be attributed to symmetrical-polarity structure which depends on the weak interaction of Vander Waals forces within particle regime.

3.3. Thermal Analysis. The thermal decomposition was studied in dry air as shown in Figure 3, and the first weight loss was observed at $300^{\circ} \mathrm{C}$ supporting the removal agglomerated organic surface impurities present in the particle. On further heating, no significant weight loss was observed supporting that the crystallinity of particle takes place at $880^{\circ} \mathrm{C}$. DSC curve shows an endothermic peak at $860^{\circ} \mathrm{C}$, which supports the formation of crystalline particles. An exothermic peak at $920^{\circ} \mathrm{C}$ supports the crystallinity with phase-chemical purity. It was observed that the morphologies of nanopowder can be controlled with the help of reaction methodology and type of ionic solvents used [12]. The soft-chemical approach is attracting more attention, because it is easy to understand the chemistry involved in designing advanced nanomaterials from molecular precursor.

3.4. X-Ray Diffraction Studies. X-ray diffraction pattern of the as-synthesized cobalt oxide nanopowder was analyzed to investigate the phase structure along with its crystallinity as

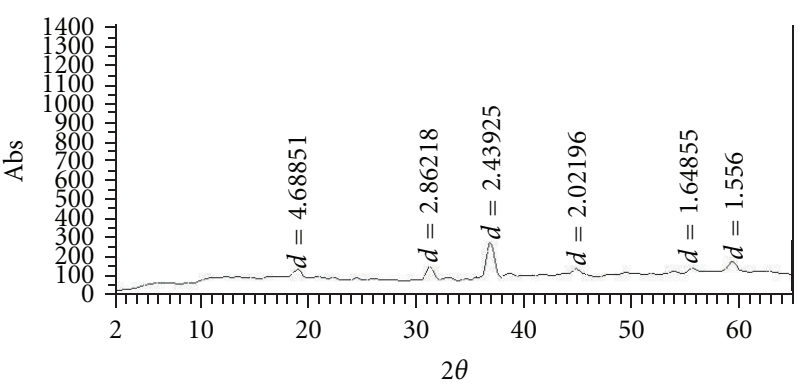

FIGURE 4: XRD pattern for cobalt oxide nanoparticles after calcination at $450^{\circ} \mathrm{C}$.

illustrated in Figure 4. The peaks were indexed to pure phase with a face-centered cubic structure, which corresponds to JCPDS file (43-1004) after annealing the sample and by matching Bragg reflection peaks. The average particle size calculated with the help of Debye-Scherrer equation was found to be $50 \mathrm{~nm}$. It was observed that the peak intensity increases with a narrowing down particle size distribution with high purity on calcination [13]. The EDX supports the formation of $\mathrm{CoO}$ nanoparticle with a chemical composition in the ratio of $1: 1$ as supported by TEM and SAED technique which supports the presence of good compositional homogeneity in the nanoparticles.

3.5. SEM-EDX. Figure 5 supports the microcrystalline nature of the particle after calcinations with least degree of agglomeration. Particles seem to have an irregular shape with chemical homogeneity with uniform morphology due to the presence of interparticle surface connectivity. It was observed that the annealing temperature increases the crystalline nature of the particle that changes due to nucleation $[14,15]$.

3.6. TEM. TEM images show the presence of aggregated polycrystalline shape of a particle with narrow size distribution having an irregular shape due to surface particle interaction. The particle size as calculated by using DebyeScherrer equation has been supported by TEM as highlighted in Figure 6. Some distorted strings were observed due to selfalignment orientation taking place due to the presence of weak interactions. The XRD patterns support the presence 


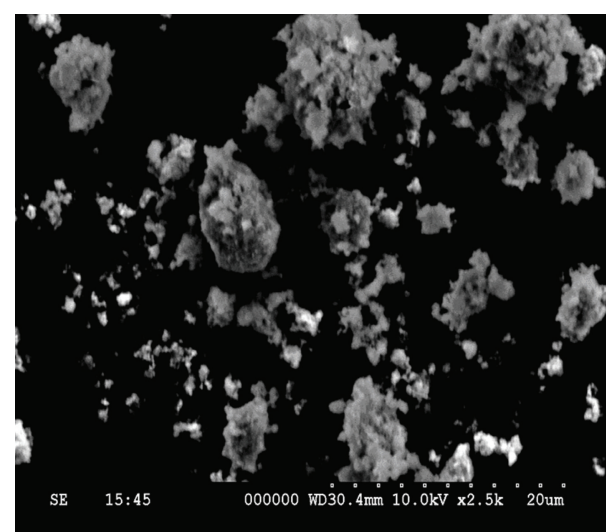

FIgURE 5: SEM images of cobalt oxide nanoparticles were taken after calcination at $450^{\circ} \mathrm{C}$ for 4 hours in the presence of inert atmosphere.

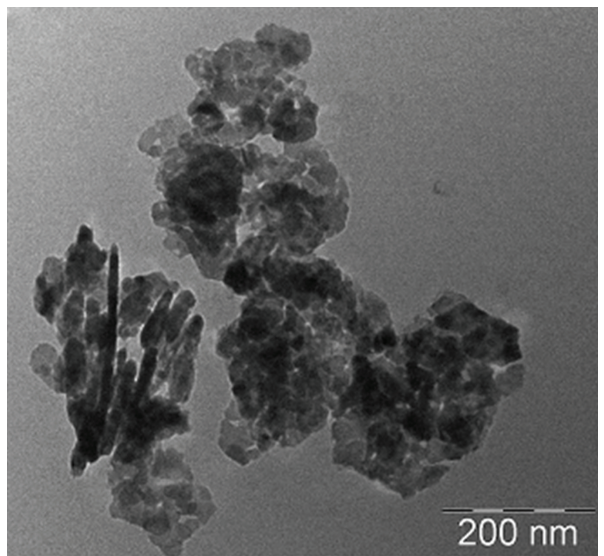

(a)

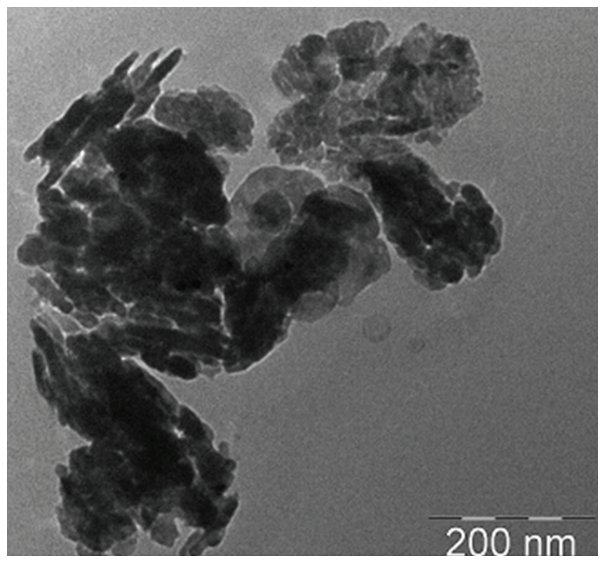

(c)

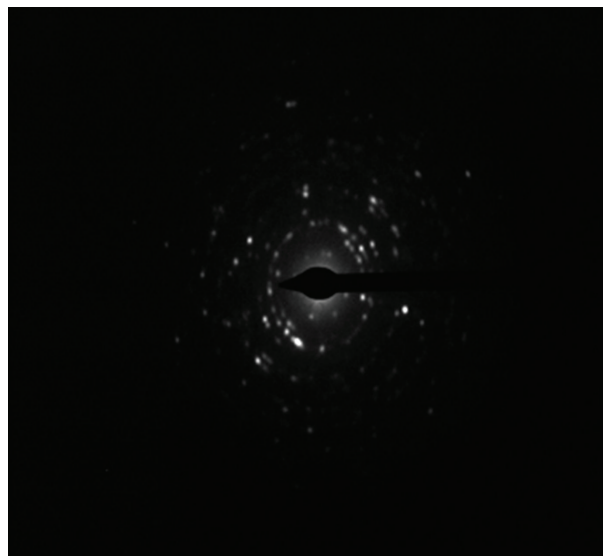

(b)

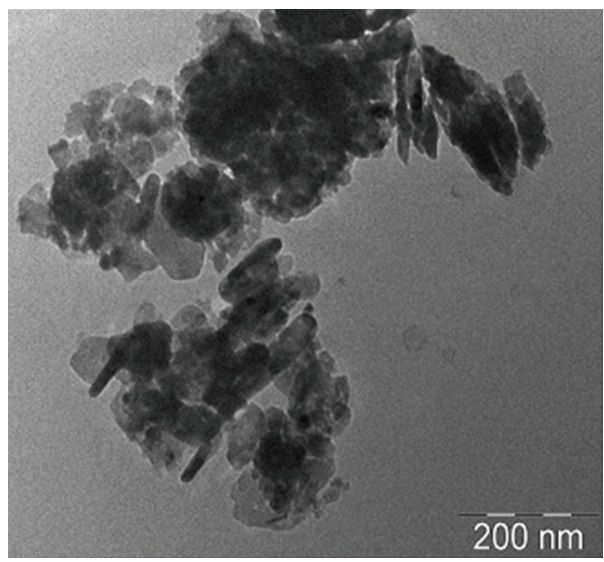

(d)

FIGURE 6: TEM images of cobalt oxide nanoparticles after calcination at $450^{\circ} \mathrm{C}$.

of crystalline phase that is in good agreement with reported values. SAED diffraction pattern shows the presence of welldefined clear spot having the polycrystalline nature of a particle [16].

3.7. BET. The surface area and pore volume were found to be $33.0715 \mathrm{~m}^{2} / \mathrm{g}$ and $7.591 \mathrm{~cm}^{3} / \mathrm{g}$, respectively, supporting the presence of limited porosity with a pore size distribution. These values support their industrial applications as sensors $[13,15]$.

\section{Conclusions}

Cobalt oxide nanoparticles were synthesized in good yield with the help of soft chemical approach with controlled size 
distribution and phase purity. This methodology is a novel, cheap, and convenient technique suitable for a large-scale production for other metal oxide nanomaterials having monomodal distribution with advanced functional properties for their future use in technological applications.

\section{References}

[1] L. Zhang and D. Xue, "Preparation and magnetic properties of pure CoO nanoparticles," Journal of Materials Science Letters, vol. 21, no. 24, pp. 1931-1933, 2002.

[2] W. Y. Li, L. N. Xu, and J. Chen, " $\mathrm{Co}_{3} \mathrm{O}_{4}$ nanomaterials in lithium-ion batteries and gas sensors," Advanced Functional Materials, vol. 15, no. 5, pp. 851-857, 2005.

[3] H. Qiao, L. Xiao, Z. Zheng, H. Liu, F. Jia, and L. Zhang, "Onepot synthesis of $\mathrm{CoO} / \mathrm{C}$ hybrid microspheres as anode materials for lithium-ion batteries," Journal of Power Sources, vol. 185, no. 1, pp. 486-491, 2008.

[4] X. Xie and W. Shen, "Morphology control of cobalt oxide nanocrystals for promoting their catalytic performance," Nanoscale, vol. 1, pp. 50-60, 2009.

[5] H. J. Guo, Q. M. Sun, X. H. Li, Z. X. Wang, and W. J. Peng, "Synthesis and electrochemical performance of $\mathrm{Co}_{3} \mathrm{O}_{4} / \mathrm{C}$ composite anode for lithium ion batteries," Transactions of Nonferrous Metals Society of China, vol. 19, no. 2, pp. 372-376, 2009.

[6] M. Salavati-Niasari, N. Mir, and F. Davar, "Synthesis and characterization of $\mathrm{Co}_{3} \mathrm{O}_{4}$ nanorods by thermal decomposition of cobalt oxalate," Journal of Physics and Chemistry of Solids, vol. 70, no. 5, pp. 847-852, 2009.

[7] V. Srinivasan and J. W. Weidner, "Capacitance studies of cobalt oxide films formed via electrochemical precipitation," Journal of Power Sources, vol. 108, no. 1-2, pp. 15-20, 2002.

[8] D. Srikala, V. N. Singh, A. Banerjee, B. R. Mehta, and S. Patnaik, "Synthesis and characterization of ferromagnetic cobalt nanospheres, nanodiscs and nanocubes," Journal of Nanoscience and Nanotechnology, vol. 9, no. 9, pp. 5627-5632, 2009.

[9] K. H. Kim and K. B. Kim, "Ultrasound assisted synthesis of nano-sized lithium cobalt oxide," Ultrasonics Sonochemistry, vol. 15, no. 6, pp. 1019-1025, 2008.

[10] A. I. Vogel, Textbook of Quantitative Chemical nalysis, Longman, London, UK, 5th edition, 1989.

[11] A. Salimi, R. Hallaj, and S. Soltanian, "Immobilization of hemoglobin on electrodeposited cobalt-oxide nanoparticles: direct voltammetry and electrocatalytic activity," Biophysical Chemistry, vol. 130, no. 3, pp. 122-131, 2007.

[12] W. W. Wang and Y. J. Zhu, "Microwave-assisted synthesis of cobalt oxalate nanorods and their thermal conversion to $\mathrm{Co}_{3} \mathrm{O}_{4}$ rods," Materials Research Bulletin, vol. 40, no. 11, pp. 1929-1935, 2005.

[13] R. Venkatnarayan, V. Kanniah, and A. Dhathathreya, Journal of Chemical Sciences, vol. 188, p. 179, 2006.

[14] Q. Yuanchun, Z. Yanbao, and W. Zhishen, "Preparation of cobalt oxide nanoparticles and cobalt powders by solvothermal process and their characterization," Materials Chemistry and Physics, vol. 110, no. 2-3, pp. 457-462, 2008.

[15] J. H. Smått, B. Spliethoff, J. B. Rosenholm, and M. Lindén, "Hierachically porous nanocrystalline cobalt oxide monoliths through nanocasting," Chemical Communications, vol. 10, no. 19, pp. 2188-2189, 2004.
[16] I. Luisetto, F. Pepe, and E. Bemporad, "Preparation and characterization of nano cobalt oxide," Journal of Nanoparticle Research, vol. 10, no. 1, pp. 59-67, 2008. 

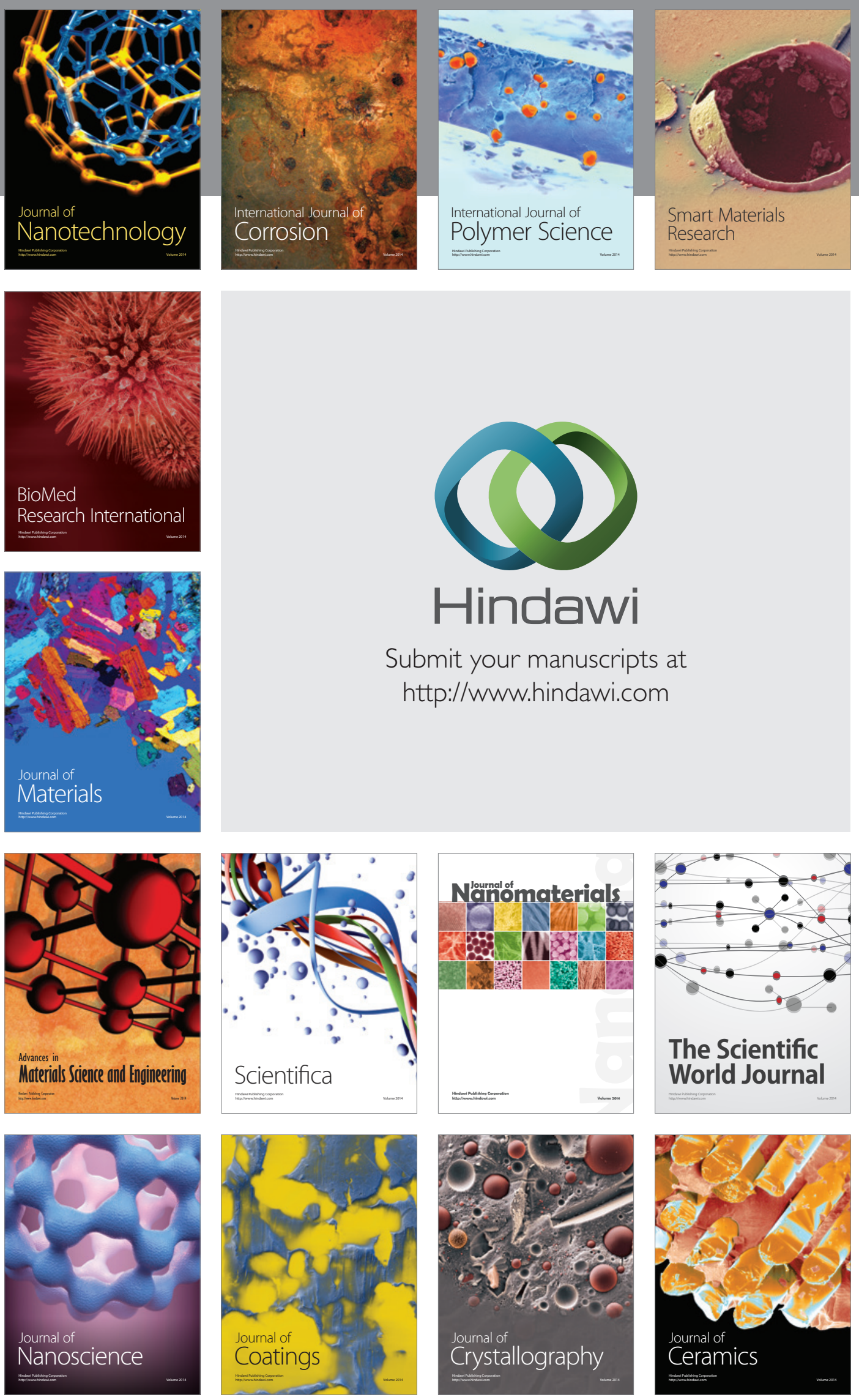

The Scientific World Journal

Submit your manuscripts at

http://www.hindawi.com

\section{World Journal}

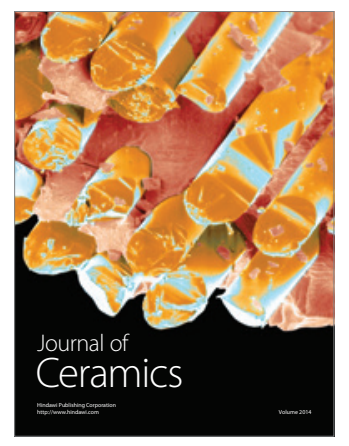

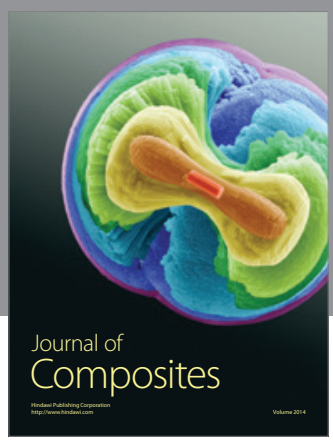
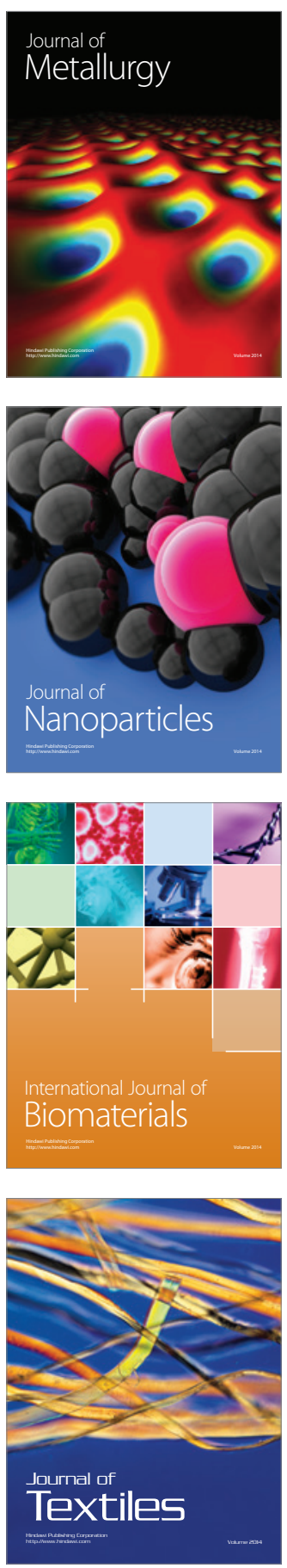